\title{
Effective slip lengths for immobilized superhydrophobic surfaces
}

\author{
Darren G. Crowdy $\dagger$ \\ (Received 18 April 2017; revised 13 June 2017; accepted 28 June 2017; \\ first published online 24 July 2017)
}

Department of Mathematics, Imperial College London, 180 Queen's Gate, London, SW7 2AZ, UK

Analytical solutions are found for both longitudinal and transverse shear flow, at zero Reynolds number, over immobilized superhydrophobic surfaces comprising a periodic array of near-circular menisci penetrating into a no-slip surface and where the menisci are no longer shear-free but are taken to be no-slip zones. Explicit formulae for the associated longitudinal and transverse effective slip lengths are derived; these are then compared with analogous results for superhydrophobic surfaces of the same characteristic geometry but where the menisci are shear-free. The new formulae give results that are consistent with recent experimental observations that have prompted suggestions that menisci that are assumed to be free of shear have in fact been immobilized. Significantly, for transverse shear flow, it is found that at critical downward meniscus protrusion angles of around $47^{\circ}$, for many surface geometries, it is impossible to distinguish, purely from the effective slip length, between a no-shear and a no-slip boundary condition. We also find that immobilized menisci bowing into the grooves at supercritical angles just below $90^{\circ}$ can be almost twice as slippery to transverse shear as no-shear menisci. The results are relevant to recent discussion as to whether surface immobilization, due to contamination by surfactants or other physical mechanisms, is compromising drag reduction properties expected from an assumed no-shear condition.

Key words: drag reduction, flow control, interfacial flows (free surface)

\section{Introduction}

Superhydrophobic surfaces are a special class of patterned surfaces on which the presence of free surface menisci spanning surface protrusions in the microstructure of the surface is presumed to supply close to no-shear regions that can enhance slip

$\dagger$ Email address for correspondence: d.crowdy@imperial.ac.uk 


\section{G. Crowdy}

(Rothstein 2010). Over the last decade, there has been huge research effort in trying to understand the slip properties of such surfaces. However, the historical development of these endeavours has been characterized by contradictory observations with large discrepancies among the slippage levels reported across the literature. A useful recent review with many of the relevant references has been given by Lee, Choi \& Kim (2016).

There is now mounting evidence that contamination by surfactants, even in trace amounts (Peaudecerf et al. 2017), can immobilize superhydrophobic surfaces, rendering them, essentially, no-slip surfaces. Kim \& Hidrovo (2012) performed experiments in a rectangular microchannel with regular sidewall patterning to visualize the location of the air-water interface within the roughness elements. One of their principal conclusions from this study of Poiseuille flow through the microchannel was that the air-water interface more closely resembles a no-slip boundary than a shear-free one, and that a Wenzel state can have better friction reducing properties than the Cassie state. In a joint experimental and numerical study of a longitudinal flow scenario, Bolognesi, Cottin-Bizonne \& Pirat (2014) tracked the effects of the meniscus shape, and the liquid-air interfacial friction properties, to examine the surface drag reduction properties. In this way, they were able to test the no-shear assumption on the menisci. These authors also suggested that their observed results were more consistent with immobilized no-slip free surfaces than those exhibiting zero shear. Bolognesi et al. (2014) suggested surfactants or other surface contaminants as possible causes for this surface immobilization. In a very recent study, Peaudecerf et al. (2017) focused on examining the effect of surfactants on hydrodynamic slip in longitudinal flows along unidirectionally patterned surfaces. Careful pressure control was used to maintain flat menisci, with the conclusion that even trace amounts of surfactants can cause a dramatic reduction in the expected slip enhancement properties of the surfaces, again, by causing an immobilization of these surfaces into effective no-slip zones.

The purpose of this paper is to offer complementary theoretical insights into the question of which surface condition is active on curved menisci in unidirectional superhydrophobic surfaces: an explicit comparison of the effective slip lengths for both no-shear and no-slip boundary conditions in longitudinal and transverse shear flows is performed. To elucidate further, an important class of superhydrophobic surfaces consists of those with a periodic unidirectional patterning of no-slip pillars separated by grooves that, in the Cassie state, are spanned by free surface menisci that trap a second fluid (e.g. air) in the subphase grooves (here, we ignore subphase fluid effects). Often, due to high pressures in the working fluid, the menisci will bow down into the grooves. Assuming, in the zero-capillary-number limit, that the menisci are shear-free, then they will be circular arcs with curvature determined by a Laplace-Young force balance. For steady longitudinal semi-infinite shear flow $(u, v, w)=(0,0, w(x, y))$ with shear rate $\dot{\gamma}$ over such surfaces, the longitudinal effective slip length $\lambda^{\|}$is defined as the quantity such that, as $y \rightarrow \infty$,

$$
w(x, y) \rightarrow \dot{\gamma}\left(y+\lambda^{\|}\right) .
$$

For a steady transverse shear flow $(u, v, 0)$, the effective transverse slip length $\lambda^{\perp}$ is such that, as $y \rightarrow \infty$, the velocity has the form

$$
(u, v, 0) \rightarrow\left(\dot{\gamma}\left(y+\lambda^{\perp}\right), 0,0\right) .
$$

It is important to note that these two effective slip lengths can be defined whatever the nature of the boundary condition imposed on the curved meniscus. If a curved 


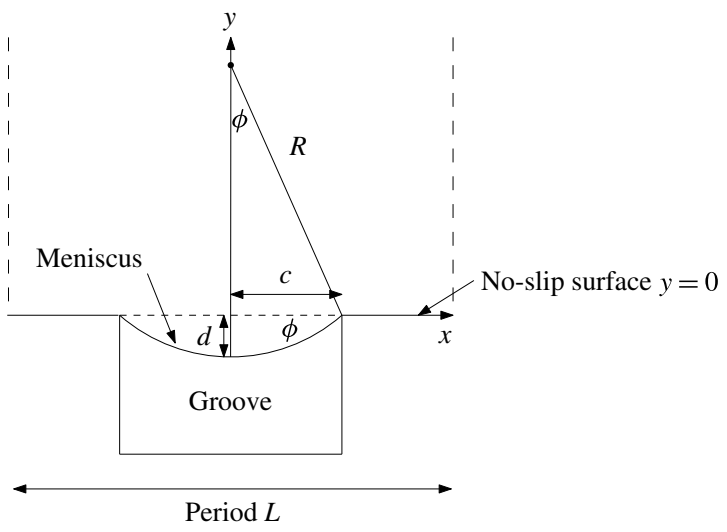

FIgURE 1. Downward protruding circular meniscus in a period- $L$ superhydrophobic surface. This paper compares effective slip lengths when the meniscus is both no-shear and no-slip.

meniscus happens to be a no-slip surface - that is, it has been immobilized by some means - then we are equivalently dealing with a corrugated no-slip wall with circulararc 'riblets', and it is more common then to refer to these effective slip lengths as protrusion heights (Bechert \& Bartenwerfer 1989; Luchini, Manzo \& Pozzi 1991). In the latter context, since the choice of the $y$-origin is arbitrary, the only physically significant quantity is the difference $\lambda^{\|}-\lambda^{\perp}$, which is independent of the choice of $y$-origin, and quantifies how much a corrugated no-slip wall impedes a transverse shear relative to a longitudinal shear.

We now summarize our results. Consider the unidirectional superhydrophobic surface whose period window of length $L$ is shown in figure 1: a meniscus, of width $2 c$, protrudes into the groove by angle $\phi$ (here, we will not consider menisci protruding above $y=0$ ). On introducing the usual complex variable $z=x+\mathrm{i} y$, we introduce a family of approximating surfaces described by the image of a unit $\zeta$-circle in a parametric $\zeta$-plane under the conformal map

$$
z=x+\mathrm{i} y=z(\zeta)=-\frac{\mathrm{i} L}{2 \pi} \log \zeta+\frac{a}{\zeta-\zeta_{0}}-\frac{a}{1-\zeta_{0}},
$$

with parameters $a$ and $\zeta_{0}$ given in terms of $c, L$ and $\phi$ as

$$
\left.\begin{array}{c}
\zeta_{0}=\left[\frac{2 c}{L} \cot (\phi / 2)\left(\phi \operatorname{cosec}^{2} \phi-\cot \phi-\frac{\pi}{4} \tan ^{2}(\phi / 2)\right)-1\right]^{-1}, \\
a=\frac{\mathrm{i} c \tan (\phi / 2)}{2}\left(1-\zeta_{0}^{2}\right) .
\end{array}\right\}
$$

Clearly, $\zeta_{0}$ is real and $a$ is purely imaginary. This class of mappings is found to give images of the unit $\zeta$-disc that are very close to the semi-infinite period window with the same values of $c, L$ and $\phi$ as shown in figure 1 . Figure 2 shows the image of the map (1.3) for $\phi=90^{\circ}$ and the values $c=d=0.1,0.3,0.5$ together with superpositions of actual semicircular arcs separated by straight lines along $y=0$ characterized by the same geometry. The domains are almost indistinguishable in all cases, with just mild smoothing out of the corner points. This is found to be the case for all choices of the parameters $c, L$ and $\phi$. 


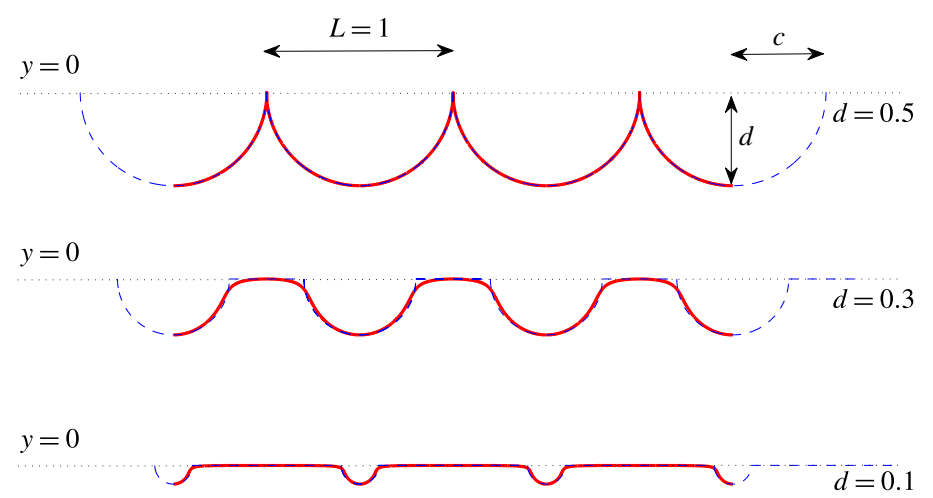

FIgURE 2. Surface shapes with period $L=1$ described by (1.3) and (1.4) for $c=d=$ $0.5,0.3$ and 0.1 (this is the case $\phi=90^{\circ}$; other protrusion angle choices with $c \neq d$ can be taken). The menisci are very close to being semicircular with near-flat inter-groove spacing of length approximately $L-2 d$. The broken lines show exactly semicircular menisci of radius $d$ separated by straight lines along $y=0$; these broken lines are barely visible except near mildly smoothed-out corners, indicating the accuracy of the approximation.

The significance of this observation is that, for the special class of surfaces (1.3) approximating the superhydrophobic surface geometry, we are able to solve explicitly for both longitudinal and transverse shear flow over these surfaces if the menisci are taken to be no-slip surfaces. Consequently, we derive the following analytical slip length formulae:

$$
\begin{gathered}
\frac{\lambda^{\|}}{c}=\frac{c}{L}\left[\phi \operatorname{cosec}^{2} \phi-\cot \phi-\frac{\pi}{4} \tan ^{2}(\phi / 2)\right], \\
\frac{\lambda^{\perp}}{c}=\frac{\tan (\phi / 2)}{8 \zeta_{0}}\left\{3+4 \zeta_{0}+\zeta_{0}^{2}+\frac{1}{\pi} \frac{\left(1-\zeta_{0}^{2}\right)^{2}}{\left[2(c / L) \zeta_{0} \tan (\phi / 2)+(1 / \pi)\left(1-\zeta_{0}^{2}\right)\right]}\right\},
\end{gathered}
$$

with $\zeta_{0}$ given in terms of the geometrical parameters $c, L$ and $\phi$ by (1.4). We expect (1.5) and (1.6) - which are the exact effective slip lengths for the approximating surfaces - to give excellent approximations to the effective slip lengths for shear flow over the no-slip circular-arc geometries of figure 1. On a physical note, it is by no means clear that the actual shape of a steady immobilized meniscus will be a circular arc - this will be a function of precisely what physical mechanism is immobilizing it (e.g. surfactant or thermally induced Marangoni stresses, transition to a Wenzel state) - but, for purposes of comparing effective slip lengths with the zero-capillary-number limit of a no-shear meniscus, we proceed under this assumption.

With formulae (1.5) and (1.6) to hand, we then compare them with analogous results for longitudinal and transverse shear flows over the same surfaces but where the menisci are now shear-free; the required results already exist in the literature. (Concerning the arbitrary choice of $y$-origin just discussed, since we compare the quantities $\lambda^{\|}$and $\lambda^{\perp}$ for the same surface geometry but two different choices of boundary condition, the results have physical significance in quantifying the difference between the effective slip properties for shear flow over surfaces with these two boundary condition choices.) The implications of these new results for possible immobilization of superhydrophobic surfaces by impurities or surfactant contamination are examined in $\S 5$. 

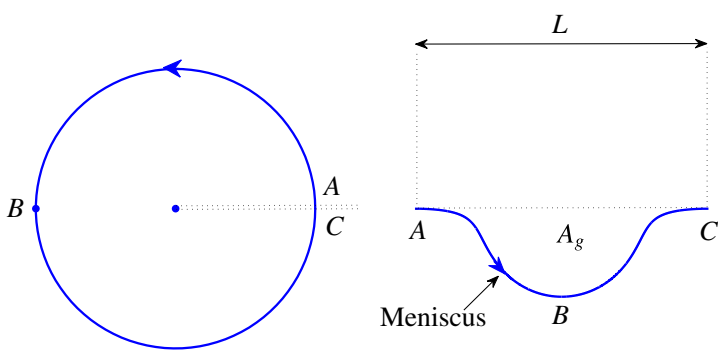

FIGURE 3. Conformal mapping from the unit $\zeta$-disc with logarithmic branch cut to a single period of the surface. Points labelled by the same letter correspond under the mapping (1.3). The area $A_{g}$ beneath $y=0$ is also indicated. Point $A$ in the physical plane is at $z=0$.

\section{A special class of surfaces}

The class of surfaces to be studied will be described mathematically by conformal mappings (1.3) from the cut unit disc in a complex parametric $\zeta$-plane to a single period window of the flow pattern over the $L$-periodic surface (figure 3 ). We need $\left|\zeta_{0}\right|>1$ so that the mapping avoids a simple pole inside the unit $\zeta$-disc. Other constraints on $a$ and $\zeta_{0}$ arise from the need to ensure univalency of the mapping. The branch of the logarithm (the two sides of the branch cut represent the two period window edges) is chosen to be along the positive real $\zeta$-axis. The preimage of infinity is $\zeta=0$. The constant term in (1.3) ensures that $z(1)=0$. It should be noted that $A_{g}$, defined as the fluid area below $y=0$, is

$$
A_{g}=\frac{1}{2 \mathrm{i}} \oint_{|\zeta|=1} \bar{z}(1 / \zeta) z^{\prime}(\zeta) \mathrm{d} \zeta+\frac{1}{2 \mathrm{i}} \int_{L}^{0} z \mathrm{~d} z=L \operatorname{Im}\left[\frac{a}{1-\zeta_{0}}+\frac{a}{\zeta_{0}}\right]+\frac{\pi|a|^{2}}{\left(1-\left|\zeta_{0}\right|^{2}\right)^{2}}
$$

Suppose now that we seek maps within this class that are 'close' to a circular-arc groove of length $2 c$ with protrusion angle downwards $\phi$. The geometry is clearly parametrized by $c$ and $\phi$, but we now seek the corresponding mapping parameters $a$ and $\zeta_{0}$. From simple geometrical considerations, it can be shown that

$$
R=\frac{c}{\sin \phi}, \quad d=R-R \cos \phi=c \tan (\phi / 2), \quad A_{g}=\frac{c^{2}}{\sin ^{2} \phi}(\phi-\sin \phi \cos \phi) . \quad(2.2 a-c)
$$

To construct the class of mappings (1.3)-(1.4), we now insist that the image of $\zeta=$ $\mathrm{e}^{\mathrm{i} \pi}=-1$ under (1.3) is at $L / 2-\mathrm{i} d$, with $d$ given as in (2.2), and that the area $A_{g}$ beneath the surface is given by the formula in (2.2). This leads, on use of (2.1) and (2.3), to

$$
\zeta_{0}=\left[\frac{2}{d L}\left(A_{g}-\frac{\pi d^{2}}{4}\right)-1\right]^{-1}, \quad a=\frac{\mathrm{i} d}{2}\left(1-\zeta_{0}^{2}\right) .
$$

On substituting for $A_{g}$ and $d$ from (2.2), where they are given as functions of $c$ and $\phi$, we arrive after some algebra at (1.4). While the boundary shapes encoded in the class of conformal mappings (1.3)-(1.4) are approximations to the circulararc boundaries in figure 1, by construction, the area $A_{g}$ beneath the $y=0$ surface is exactly the same for both - an important fact given that, for longitudinal flow 


\section{G. Crowdy}

with a no-slip boundary condition, any effective slip is intuitively associated with changes in the cross-sectional area of the flow. Figure 2 gives evidence of how closely these mappings approximate downward protruding circular arcs. One should note the serendipity of this: we have no right to expect, a priori, that the fitting of just two parameters will produce conformal mappings with images that are uniformly close to the desired target curve. That it happens to be successful is pure mathematical luck.

\section{Longitudinal flow}

Assuming no driving pressure gradient, the longitudinal flow $w(x, y)$ satisfies

$$
\nabla^{2} w=0
$$

in the fluid region above the surface, so it is natural to introduce the complex potential $($ Crowdy 2011b) $h(z)=\chi+\mathrm{i} w$. Since $w \rightarrow \dot{\gamma} y$ as $|z| \rightarrow \infty$, then we require

$$
h(z) \rightarrow \dot{\gamma} z
$$

as $z \rightarrow \infty$ with the no-slip condition

$$
w=\operatorname{Im}[h(z)]=0, \text { on the surface. }
$$

Now, consider the function

$$
H(\zeta) \equiv h(z(\zeta))=-\frac{\mathrm{i} \dot{\gamma} L}{2 \pi} \log \zeta .
$$

It can be directly verified that this function satisfies all requirements. Since, from (1.3), we can write

$$
-\frac{\mathrm{i} L}{2 \pi} \log \zeta=z-\frac{a}{\zeta-\zeta_{0}}+\frac{a}{1-\zeta_{0}}
$$

then, on comparison with (1.1), the slip length $\lambda^{\|}$can be determined as

$$
\lambda^{\|}=\operatorname{Im}\left[\frac{a}{1-\zeta_{0}}+\frac{a}{\zeta_{0}}\right]=\frac{d}{2}\left(1+\frac{1}{\zeta_{0}}\right) .
$$

On use of (1.4) and (2.2) to substitute for $d$ and $\zeta_{0}$ in terms of $c$ and $\phi$, we arrive at (1.5).

\section{Transverse flow}

The transverse flow problem is more difficult, not least because it is not conformally invariant, but, remarkably, it is exactly solvable for domains described by conformal mappings of the form (1.3), as we now show. Our mathematical derivation of the transverse flow solution is similar in spirit to that of Richardson (1973), but we have eschewed his approach based on Taylor series, since, for our class of solutions (1.3), it would require an infinite number of terms. We adopt a more function theoretic approach.

The streamfunction $\psi(x, y)$ associated with a two-dimensional Stokes flow of a fluid of viscosity $\mu$ satisfies the biharmonic equation (Langlois 1964; Crowdy 2011a)

$$
\nabla^{4} \psi=0
$$

With $z=x+\mathrm{i} y$, the general solution to (4.1) is available in the form

$$
\psi(x, y)=\operatorname{Im}[\bar{z} f(z)+g(z)],
$$


Effective slip lengths for immobilized superhydrophobic surfaces

where $f(z)$ and $g(z)$ are two analytic functions in the fluid region. Let

$$
(u, v)=(\partial \psi / \partial y,-\partial \psi / \partial x)
$$

denote the components of the fluid speed and let $p$ denote the fluid pressure. It can be shown (Langlois 1964; Crowdy 2011a) that

$$
\frac{p}{\mu}-\mathrm{i} \omega=4 f^{\prime}(z), \quad u-\mathrm{i} v=-\overline{f(z)}+\bar{z} f^{\prime}(z)+g^{\prime}(z), \quad \omega=\frac{\partial v}{\partial x}-\frac{\partial u}{\partial y}, \quad(4.4 a-c)
$$

where primes denote differentiation with respect to the argument of the function. For the required shear flow as $y \rightarrow \infty$, we need

$$
f(z) \rightarrow \frac{\mathrm{i} \dot{\gamma} z}{4}+\text { const., } \quad g^{\prime}(z) \rightarrow-\frac{\mathrm{i} \dot{\gamma} z}{2}+\text { const. }
$$

Henceforth, we take $\dot{\gamma}=1$. It is convenient to define the Schwarz function (Davis 1974)

$$
\begin{aligned}
S(z) \equiv \bar{z}(1 / \zeta) & =-\frac{\mathrm{i} L}{2 \pi} \log \zeta+\frac{\bar{a} \zeta}{1-\zeta \zeta_{0}}-\frac{\bar{a}}{1-\zeta_{0}} \\
& =z(\zeta)-\frac{a}{\zeta-\zeta_{0}}+\frac{a}{1-\zeta_{0}}+\frac{\bar{a} \zeta}{1-\zeta \zeta_{0}}-\frac{\bar{a}}{1-\zeta_{0}}
\end{aligned}
$$

where we have used (1.3); hence, $S(z)=\bar{z}$ on the meniscus. Now, we define

$$
F(\zeta) \equiv f(z(\zeta)), \quad G(\zeta) \equiv g^{\prime}(z(\zeta))
$$

It will be shown that a class of explicit solutions is given by

$$
F(\zeta)=\frac{\mathrm{i} z(\zeta)}{4}+\frac{F_{1}}{\zeta-\zeta_{0}}, \quad G(\zeta)=-\frac{\mathrm{i} z(\zeta)}{4}-S(z) f^{\prime}(z)+\frac{G_{-1}}{\zeta-1 / \zeta_{0}}+G_{0}+\frac{G_{1}}{\zeta-\zeta_{0}},
$$

where $F_{1}, G_{0}, G_{1}$ and $G_{-1}$ are constants to be determined. Two of these are related by

$$
G_{-1}=-\frac{\bar{a}}{\zeta_{0}^{2}}\left[\frac{\mathrm{i}}{4}-\frac{F_{1} \zeta_{0}^{2}}{\left(1-\zeta_{0}^{2}\right)^{2} z^{\prime}\left(1 / \zeta_{0}\right)}\right] .
$$

To understand why (4.7) is the required solution, it should be noted first that the function $S(z) f^{\prime}(z)$ appearing in $G(\zeta)$ can be written as a function of $\zeta$ on use of (4.6) and the chain rule $f^{\prime}(z)=F^{\prime}(\zeta) / z^{\prime}(\zeta)$. Condition (4.9) removes the simple pole of $G(\zeta)$ due to the term $-S(z) f^{\prime}(z)$ at $\zeta=1 / \zeta_{0}$, which is inside the unit disc. Both $F(\zeta)$ and $G(\zeta)$ are then analytic in the unit $\zeta$-disc except for singularities at $\zeta=0$ which are chosen to ensure that the far-field conditions (4.5) are satisfied. Since, from (4.6), $S(z) \rightarrow z+$ const. as $|z| \rightarrow \infty$, then it is easy to check that $F(\zeta)$ and $G(\zeta)$ satisfy (4.5) as $\zeta \rightarrow 0$. The corresponding (complex) velocity field is

$$
\begin{aligned}
u-\mathrm{i} v= & \frac{\overline{\mathrm{i} z(\zeta)}}{4}-\frac{\overline{F_{1}}}{\bar{\zeta}-\zeta_{0}}+[\overline{z(\zeta)}-S(z)]\left[\frac{\mathrm{i}}{4}-\frac{F_{1}}{\left(\zeta-\zeta_{0}\right)^{2}} \frac{1}{z^{\prime}(\zeta)}\right] \\
& -\frac{\mathrm{i} z(\zeta)}{4}+\frac{G_{-1}}{\zeta-1 / \zeta_{0}}+G_{0}+\frac{G_{1}}{\zeta-\zeta_{0}}
\end{aligned}
$$




\section{G. Crowdy}

which can be shown to be $L$-periodic. On the surface where $|\zeta|=1$, and on use of (4.6), this becomes

$$
\begin{aligned}
u-\mathrm{i} v= & \left\{-\frac{\mathrm{i} a}{4}+G_{1}\right\} \frac{1}{\zeta-\zeta_{0}}+\left\{-\frac{\mathrm{i} \bar{a}}{4 \zeta_{0}{ }^{2}}+\frac{\overline{F_{1}}}{\zeta_{0}{ }^{2}}+G_{-1}\right\} \frac{1}{\zeta-1 / \overline{\zeta_{0}}} \\
& +\left\{\frac{\overline{F_{1}}}{\zeta_{0}}+G_{0}-\frac{1}{2} \operatorname{Im}\left[\frac{a}{1-\zeta_{0}}\right]-\frac{\mathrm{i} \bar{a}}{4 \zeta_{0}}\right\}
\end{aligned}
$$

We can therefore satisfy the no-slip condition everywhere on the surface if we make all of the curly-bracketed terms vanish. This implies the conditions

$$
-\frac{\mathrm{i} a}{4}+G_{1}=0, \quad-\frac{\mathrm{i} \bar{a}}{4 \zeta_{0}^{2}}+\frac{\overline{F_{1}}}{\zeta_{0}^{2}}+G_{-1}=0, \quad-\frac{1}{2} \operatorname{Im}\left[\frac{a}{1-\zeta_{0}}\right]-\frac{\mathrm{i} \bar{a}}{4 \zeta_{0}}+\frac{\overline{F_{1}}}{\zeta_{0}}+G_{0}=0
$$

These three equations, together with (4.9), determine the four constants $F_{1}, G_{-1}, G_{0}$ and $G_{1}$. On elimination of $G_{-1}$ between (4.9) and $(4.12 b)$, we find

$$
F_{1}=\frac{\mathrm{i} a D}{2(a-D)}, \quad D=-a-\frac{\mathrm{i} L}{2 \pi \zeta_{0}}\left(1-\zeta_{0}^{2}\right)^{2}
$$

With $F_{1}$ thus determined, the parameters $G_{-1}, G_{0}$ and $G_{1}$ follow explicitly from (4.9) and (4.12). Finally, the slip length is obtained by analysis of expression (4.10) as $\zeta \rightarrow$ 0 and comparison with (1.2):

$$
\lambda^{\perp}=\frac{\overline{F_{1}}}{\zeta_{0}}+\operatorname{Im}\left[\frac{a}{1-\zeta_{0}}\right]-\frac{\mathrm{i} a}{2 \zeta_{0}} .
$$

After some algebra, on use of expression (4.13) for $F_{1}$, we arrive at (1.6).

\section{Comparison of no-slip and no-shear assumptions}

Since the consensus in much previous work has been that the menisci in superhydrophobic surfaces are close to shear-free, there has been great effort in seeking to quantify the effective slip properties under a shear-free assumption. Those prior results, coupled with the complementary results derived here for the same class of superhydrophobic surface geometries, allow us to test this assumption. For longitudinal flow, Crowdy (2016) has derived a formula for the effective slip length for exactly circular no-shear menisci. The effective slip length in (1.4) of Crowdy (2016) is plotted as a function of $\phi$ in figure 4 along with the new slip length result (1.5) for a no-slip surface with the same fixed value of $2 c / L=0.5$, corresponding to the no-shear fraction used in the experiments of Bolognesi et al. (2014). (Formula (1.4) of Crowdy (2016) is known to be accurate to within 1\%-2\% for this normalized groove width.) An immediate observation is that, as $\phi$ increases, the slip associated with a no-shear surface decreases while the slip associated with a no-slip surface increases. In two experiments, referred to as M1 and M2, Bolognesi et al. (2014) observed that a more depressed groove associated with experiment M1 is associated with a larger slip length than experiment M2, where the interface invaded the groove to a lesser degree. As those authors pointed out, this is precisely not what is expected if the meniscus is a no-shear surface. Bolognesi et al. (2014) went on to suggest that the observed behaviour is more consistent with the meniscus being a no-slip surface. Figure 4 provides theoretical evidence to support this. 


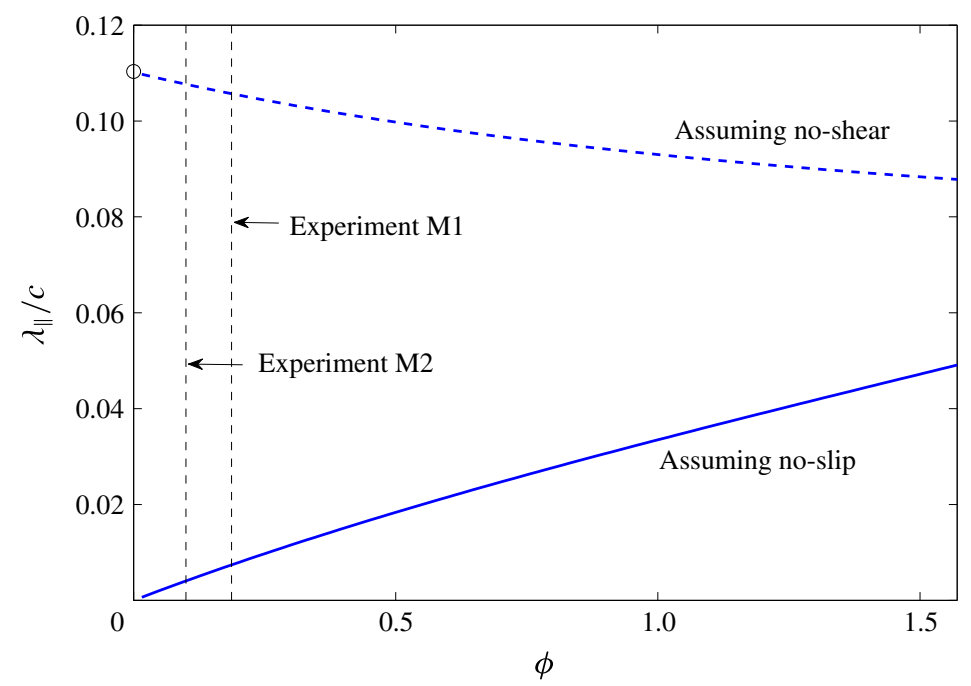

FIGURE 4. Normalized longitudinal slip length for grooves of aspect ratio $2 c / L=0.5$ as a function of downward protrusion angle $\phi$. The no-shear results are based on a slip length formula of Crowdy (2016); the no-slip results are from (1.5). Data points for $\phi=0.1870$ and $\phi=0.1$ corresponding to estimates of the meniscus geometries of experiments M1 and M2 of Bolognesi et al. (2014) are shown. The no-slip results show behaviour consistent with the experiments.

From figure 3 of Bolognesi et al. (2014), we fitted our theoretical profiles to the experimental meniscus profiles by visually estimating the geometrical parameters $d / c=1.5 \mu \mathrm{m} / 16 \mu \mathrm{m}$ for experiment $\mathrm{M} 1$ and $d / c=0.8 \mu \mathrm{m} / 16 \mu \mathrm{m}$ for experiment M2. On use of (2.2), the associated downward protrusion angles $\phi$ can be calculated, and these are indicated by the two dotted ordinates shown in figure 4. It is found that the slip associated with M1 is approximately $80 \%$ greater than that associated with M2. Bolognesi et al. (2014) reported an increase of $40 \%$, but full quantitative agreement cannot be expected since the experiments were carried out in a closed geometry, while our formula (1.5) does not take a no-slip upper wall into account. Moreover, the experimentally observed menisci display flattened bottoms, while the results here are for near-circular arcs - a fact that reminds us of the comment made earlier that it is not clear what the true shape of any immobilized meniscus will actually be. Nevertheless, the results here are in corroborative qualitative agreement with the experimental observations. Bolognesi et al. (2014) compared their observed global slip length against the prediction of a flat-meniscus no-shear result due to Philip (1972) and noticed that the former is approximately a tenth of that predicted by the no-shear model. It is clear from the ordinates drawn on figure 4 that the slip length according to the no-slip model is close to $10 \%$ of that predicted by a no-shear model, in broad agreement with the experiments (Bolognesi et al. 2014).

The results for the transverse flow case are also revealing. Figure 5 shows results for $2 c / L=0.2$, which has been deliberately chosen to be sufficiently small that we can make use of the dilute limit slip length formula found by Davis \& Lauga (2009). Figure 5 shows the predictions of this no-shear model for an exactly circular arc together with the results from the new formula (1.6) for no-slip surfaces of very nearly the same shape. Once again, we find that the slip length for a no-slip 


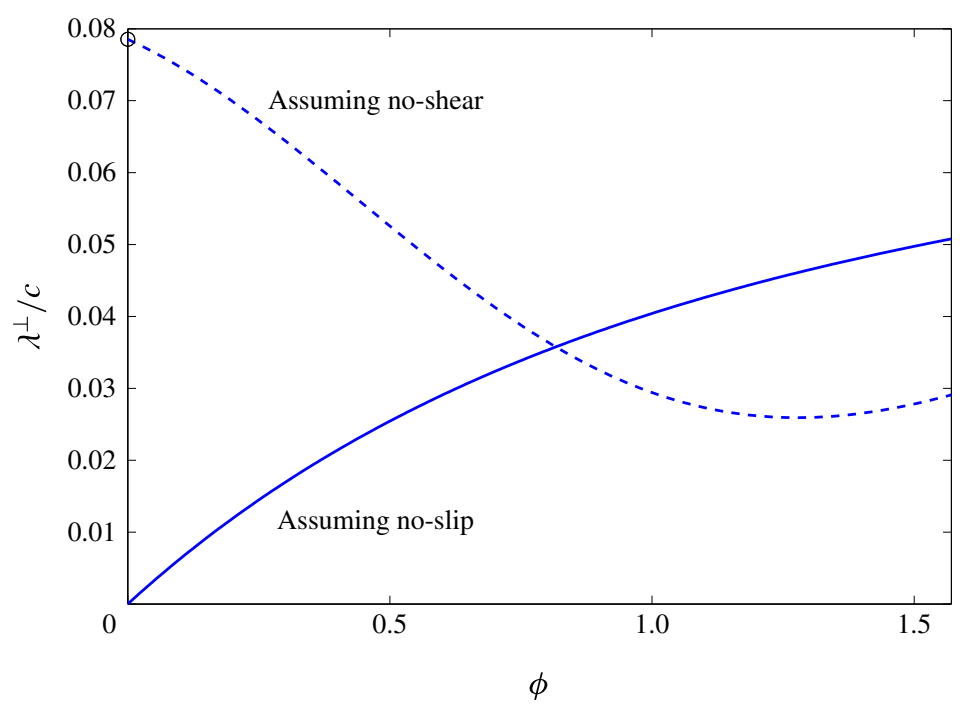

FIGURE 5. Normalized transverse slip length for grooves of aspect ratio $2 c / L=0.2$ as a function of downward protrusion angle $\phi$. The no-shear results are based on the slip length formula of Davis \& Lauga (2009); the no-slip results are from (1.6). Cross-over occurs at a critical angle of $\phi \approx 46.8^{\circ}$

surface increases with the downward protrusion angle $\phi$, in contrast to the decrease in slip length under the assumption that the menisci are shear-free. A new feature, not observed for longitudinal flow, is that we find a critical downward protrusion angle $\phi \approx 0.816 \approx 46.8^{\circ}$ at which the effective slip length associated with a no-shear surface exactly coincides with that associated with a no-slip surface. This means that, for this critical surface geometry, it is impossible to tell the nature of the boundary condition on the surface by looking at the effective slip length. Expressed differently, close to such critical meniscus geometries, one might believe that a no-shear boundary condition is active on the interface, and is thus responsible for enhanced slip, but really the enhanced slip is caused by the downward deformation of an immobilized no-slip meniscus. Indeed, albeit with respect to longitudinal flows, Bolognesi et al. (2014) made the comment 'Discriminating between the effects of a deformed meniscus, on one side, and the interfacial friction at the liquid-air interface, on the other side, is not an easy task as this requires measuring the flow nearby the meniscus while simultaneously determining its shape and position'. Our theoretical results here underline this concern: the crossing of graphs in figure 5 provides evidence of a source of possible error in interpreting experimental results based purely on effective slip data. Figure 5 shows that for angles well above critical, a no-slip meniscus can be significantly more slippery than a no-shear surface. Indeed, by changing the normalized groove width $2 c / L$ in the interval $[0,0.47]$ (where Teo \& Khoo (2016) have shown the formula of Davis \& Lauga (2009) to be $10 \%$ accurate), we find that for downward protrusion angles around $80^{\circ}$, the surface can be almost twice as slippery if immobilized as if it is free of shear (cf. Kim \& Hidrovo 2012).

In confined longitudinal microchannel flows with a lower superhydrophobic wall, say, the effective slip length is usually defined by equating the pressure-driven flux through the channel with that in a channel having a Navier slip condition, $\lambda_{N S} \mathrm{~d} w / \mathrm{d} y=$ $w$, imposed on the lower wall; the value of $\lambda_{N S}$ for which these fluxes are equal 


\section{Effective slip lengths for immobilized superhydrophobic surfaces}

(for the same driving pressure gradient) is the effective slip length. It is easy to show that for channels whose height is large compared with the pitch of the surface, this effective slip length $\lambda_{N S}$ coincides, at leading order in an aspect ratio expansion, with the effective slip lengths found here.

Finally, a different interpretation of our results is that we have found new analytical solutions for simple shear over a periodic array of near-circular surface notches (or 'riblets') in the Wenzel state with fully penetrated grooves and no free surfaces (Bechert \& Bartenwerfer 1989; Luchini et al. 1991).

\section{Acknowledgements}

The author is supported by an EPSRC Established Career Fellowship (EP/K019430/ 10), EPSRC Grant EP/K041134/1 and a Royal Society Wolfson Research Merit Award.

\section{References}

Bechert, D. W. \& BARTEnWERfER, M. 1989 The viscous flow on surfaces with longitudinal ribs. J. Fluid Mech. 206, 105-129.

Bolognesi, G., Cottin-Bizonne, C. \& Pirat, C. 2014 Evidence of slippage breakdown for a superhydrophobic microchannel. Phys. Fluids 26, 082004.

Crowdy, D. G. $2011 a$ Frictional slip lengths for unidirectional superhydrophobic grooved surfaces. Phys. Fluids 23, 072001.

CROWDY, D. G. $2011 b$ Slip length for longitudinal shear flow over a dilute periodic mattress of protruding bubbles. Phys. Fluids 22, 121703.

CROWDy, D. G. 2016 Analytical formulae for longitudinal slip lengths over unidirectional superhydrophobic surfaces with curved menisci. J. Fluid Mech. 791, R7.

Davis, A. M. J. \& LAugA, E. 2009 Geometric transition in friction for flow over a bubble mattress. Phys. Fluids 21, 011701.

DAVIS, P. J. 1974 The Schwarz Function and its Applications. Mathematical Association of America.

Kim, T. J. \& Hidrovo, C. 2012 Pressure and partial wetting effects on superhydrophobic friction reduction in microchannel flow. Phys. Fluids 24, 112003.

LANGlois, W. 1964 Slow Viscous Flow. Macmillan.

LEE, C., CHOI, C.-H. \& KIM, C.-J. 2016 Superhydrophobic drag reduction in laminar flows: a critical review. Exp. Fluids 57, 176-196.

Luchini, P., Manzo, F. \& Pozzi, A. 1991 Resistance of a grooved surface to parallel flow and cross-flow. J. Fluid Mech. 228, 87-109.

Peaudecerf, F. J., Landel, J. R., Goldstein, R. E. \& Luzzatto-Fegiz, P. 2017 Traces of surfactants can severely limit the drag reduction of superhydrophobic surfaces. Proc. Natl Acad. Sci. USA 114 (28), 7254-7259.

Philip, J. R. 1972 Flows satisfying mixed no-slip and no-shear conditions. Z. Angew. Math. Phys. 23, 353-372.

RichARDSON, S. 1973 On the no-slip boundary condition. J. Fluid Mech. 59, 707-719.

Rothstein, J. P. 2010 Slip on superhydrophobic surfaces. Annu. Rev. Fluid Mech. 109, 42-89.

TEO, C. J. \& KHOO, B. C. 2016 Flow past superhydrophobic surfaces containing longitudinal grooves: effects of interface curvature. Microfluid Nanofluid 9, 499-511. 